\title{
Investigation of piezoelectricity distributions in poly(vinylidene fluoride) by means of quartz- or laser-generated pressure pulses
}

\author{
R. Gerhard-Multhaupt, G. M. Sessler, and J. E. West \\ Bell Laboratories, Murray Hill, New Jersey 07974 and Institute for Electroacoustics, Technical University of \\ Darmstadt, Merckstrasse 25, D-6100 Darmstadt, Federal Republic of Germany \\ K. Holdik, M. Haardt, and W. Eisenmenger \\ Physics Institute (I), University of Stuttgart, Pfaffenwaldring 57, D-7000 Stuttgart 80, Federal Republic of \\ Germany
}

(Received 15 August 1983; accepted for publication 1 November 1983)

\begin{abstract}
The direct determination of charge, polarization, or piezoelectricity profiles in thin dielectrics is now possible if piezoelectrically generated pressure steps or pulses and laser-induced pressure pulses are employed. These recently developed high-resolution methods were applied to the same piezoelectric poly(vinylidene fluoride) (PVDF) samples. Comparison of the respective results demonstrates the feasibility, the advantages, and the limitations of the new techniques for the study of piezoelectricity distributions. It is confirmed that, for relatively low poling fields, the piezoelectric activity of thermopoled PVDF foils is often confined to a layer near the positively biased surface. The same effect is found for poling with a positive corona discharge. For high-field corona poling, the piezoelectric activity extends throughout the PVDF film.
\end{abstract}

PACS numbers: 77.60. $+\mathrm{v}, 77.30 .+\mathrm{d}, 79.20 . \mathrm{Ds}, 81.70 .+\mathrm{r}$

\section{INTRODUCTION}

Since its discovery ${ }^{1}$ in 1969 , the piezoelectricity in poly(vinylidene fluoride) (PVDF) has attracted widespread attention. Considerable efforts to further the understanding as well as the applications of this rather unique material are reflected in a number of recent review articles ${ }^{2-11}$ and in an extensive bibliography. ${ }^{12}$

Under certain poling conditions, the piezoelectric or pyroelectric activity of PVDF films becomes strongly depth dependent, ${ }^{13-16}$ This effect was originally discovered and studied on layered sample stacks. Layering made the separate measurement of the piezoelectric (or pyroelectric) response at different depths of the composite sample possible. The resolution of this technique is limited by the thickness of a single foil (between 6 and $25 \mu \mathrm{m}$ ).

A few years ago, the use of pressure waves for nondestructive charge- or field-distribution measurements was suggested. ${ }^{17,18}$ Previous implementations of this idea were based on pressure-wave generation by means of a shock tube, ${ }^{18} \mathrm{a}$ ruby laser, ${ }^{19}$ a high-voltage spark, ${ }^{20}$ and a $\mathrm{CO}_{2}$ laser. ${ }^{21}$ The direct experimental resolution of all these approaches is limited to a few tens of micrometers.

Recently, two complementary high-resolution methods (down to $1 \mu \mathrm{m}$, depending on sample properties) for the direct and nondestructive scanning of polarization (and charge) distributions in the thickness direction of thin films became available. ${ }^{22-26}$ Both techniques are based on the idea of using pressure waves: A piezoelectrically generated pressure step ${ }^{23-25}$ (PPS) leads to a uniform deformation of the sample volume behind the propagating step, whereas a laserinduced pressure pulse ${ }^{22,26,27}$ (LIPP) travels as a compression layer through the specimen. ${ }^{28}$ For the LIPP method a short-pulse Nd:YAG laser is utilized rather than the ruby and $\mathrm{CO}_{2}$ lasers previously employed. ${ }^{19.21}$
The two new methods, which have already been used separately for the investigation of Teflon FEP, ${ }^{22,26,29}$ Mylar PETP, ${ }^{22,26,29}$ and PVDF $^{23-26}$ samples, are directly compared in the present paper. This is done by studying the same PVDF samples with both techniques and comparing the results. Furthermore, some preliminary experimental results, obtained with piezoelectrically generated pressure pulses (PPP's) are included. It can be assumed that only chargecompensated polarization zones, but no net charges, are found in PVDF because of its high conductivity. ${ }^{23-25}$ Therefore, all the pressure-wave experiments reported provide a direct image either of the piezoelectricity profile in the PVDF samples or of its gradient (see below).

\section{EXPERIMENTAL METHODS}

\section{A. Samples and poling}

Two different types of commercially available PVDF were used: nominally 100- $\mu$ m-thick "Dyflor 2000" film made by Dynamit Nobel AG and nominally $12-\mu \mathrm{m}$-thick biaxially oriented capacitor-grade "KF" film made by Kureha, called Dyflor and Kureha KF, respectively. The samples were poled ${ }^{25}$ either by thermal poling ${ }^{1}$ or by means of a corona discharge ${ }^{30}$ :

For thermal poling, a circular sample of $5 \mathrm{~cm}$ diameter was placed between optically flat copper electrodes of $15 \mathrm{~mm}$ diameter and slightly pressed. A voltage of $+5 \mathrm{kV}$ was applied, and the sample was heated to $120^{\circ} \mathrm{C}$. After $1 \mathrm{~h}$ at $120^{\circ} \mathrm{C}$, the specimen was slowly cooled to room temperature with the voltage still applied. Finally, the bias was switched off. The use of pressed-on copper electrodes leads to the same results as the employment of evaporated aluminum coatings. ${ }^{31}$ 


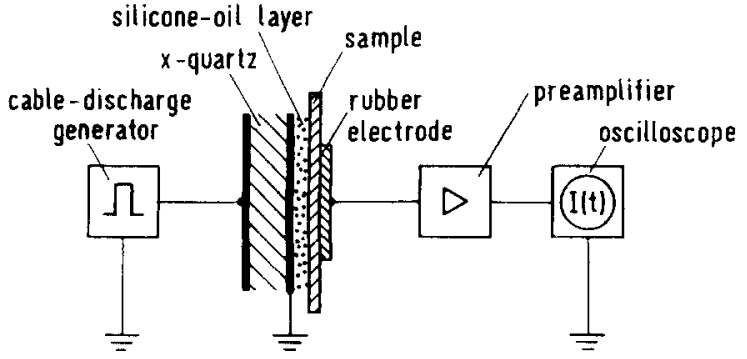

FIG, 1. Experimental setup for the piezoelectrically generated pressurestep (PPS) method. The same arrangement is used for the piezoelectrically generated pressure-pulse (PPP) technique.

For corona poling, samples of $5 \mathrm{~cm}$ diameter were placed on a grounded copper plate. A wire tip was mounted 6 or $13 \mathrm{~mm}$ above the plate and biased with about $+18 \mathrm{kV}$ for $2 \mathrm{~min}$. The resulting corona discharge from the wire tip charges the sample surface to an equivalent surface potential of more than $+10 \mathrm{kV}$. The surface-charge layer produces a strong poling field across the foil, since an image-charge layer of opposite polarity and equal charge density is formed on the grounded backplate.

\section{B. Piezoelectrically generated pressure steps (PPS's)}

\section{Experimenta/ setup}

The experimental arrangement of the PPS technique is schematically shown in Fig. 1. Square pulses of 100-ns duration from a cable-discharge generator are used to drive a piezoelectric $X$-quartz plate. The resulting pair of alternating pressure steps is coupled into the unmetallized PVDF sample and propagates through it. In a charged or poled sample, a short-circuit-current signal is generated. Further details of the method are given elsewhere. ${ }^{23-25,29}$

\section{Principle of operation}

The acoustical step wave, which consists of a compression (or rarefaction) of sample material, propagates through the sample with the velocity $c$ of longitudinal sound waves. At a given time $t$ after the step wave entered the sample, its compression extends to a depth $x=c t$. During the following time interval $\Delta t$, an additional sample layer of thickness $\Delta x=c \Delta t$ at the depth $x=c t$ becomes compressed. Thus, under short-circuit conditions, the corresponding charge $\Delta Q(t)$ flowing between the sample electrodes is given by ${ }^{32}$

$$
\frac{\Delta Q(t)}{A}=e_{33}^{*}(c t) \cdot S \frac{\Delta x}{s},
$$

where $A, e_{33}^{*}$, and $s$ are the area, the apparent piezoelectric coefficient in the thickness direction, and the thickness of the sample, respectively. The strain amplitude $S$ is determined by the particle velocity $v$ or the pressure amplitude $p$ in the step wave ${ }^{23-25}: S=-v / c=-p /\left(\rho_{0} c^{2}\right)$, where $\rho_{0}$ denotes the density of the sample. In Eq. (1), the factor $\Delta x / s$ is required, since the charge is generated directly at the layer of thickness $\Delta x$ whereas the measurement of $\Delta Q(t)$ is taken at the sample electrodes of distance $s$.

Using $\Delta Q=I \cdot \Delta t$ ( $I$ is the short-circuit current) and
$\Delta x=c \cdot \Delta t$, and dividing Eq. (1) by $\Delta t$, one obtains $\mathrm{s}^{2, \ldots .25}$

$$
I(t)=-\frac{A}{s} v e_{33}^{*}(c t)=-\frac{A}{s} \cdot \frac{p}{\rho_{0} c} e_{33}^{*}(c t) .
$$

According to this equation, the short-circuit current response to a pressure-step experiment provides a direct image of the spatial distribution of the apparent piezoelectricity in the sample.

\section{Calibration}

The PPS experiment can be calibrated by means of Eq. (2) if the particle velocity $v$ of the step wave in the sample is calculated from the acoustic transmission ratio between quartz and sample material ${ }^{23,33}$ :

$$
v=\frac{2 \rho_{Q} c_{Q}}{\rho_{Q} c_{Q}+\rho_{0} c} v_{Q},
$$

where $\rho_{Q}$ and $c_{Q}$ are the density and the sound velocity in quartz, respectively. The particle velocity $v_{Q}$ in the $X$-quartz plate is determined by the properties of the quartz and by the voltage amplitude of the square pulse. ${ }^{33,34}$ Thus, a calibrated ordinate can be calculated for all the figures presenting PPS responses.

This theoretical calibration is checked by using another quartz plate in place of the sample. A typical result of such an experiment is depicted in Fig. 2. From the quartz response, the calibration factor $A v / s$ can be determined independently. Agreement between the experimental calibration and the above theoretical method is very good. ${ }^{25}$ The quartz response shown in Fig. 2 represents a direct image of the pressure step ${ }^{3,34}$ and demonstrates its short rise time of approximately $1 \mathrm{~ns}$.

\section{Laser-induced pressure pulses (LIPP's)}

\section{Experimental setup}

Figure 3 depicts the experimental arrangement of the LIPP method as used here. One surface of a two-sided metallized sample is coated with a thin $(2-4 \mu \mathrm{m})$ graphite layer which is illuminated by a laser-light pulse of 70-ps duration and about $10-\mathrm{mJ}$ energy (corresponding to an average pulse power of about $0.14 \mathrm{GW}$ ). The ablation, which originates from heating of the graphite target, generates a recoil, the temperature gradient in the graphite causes thermal stresses, and the electromagnetic radiation pressure produces an additional recoil. Together, these effects launch the desired pressure pulse that propagates through the sample with the

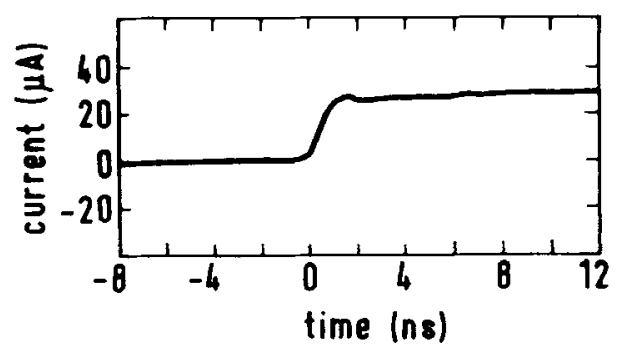

FIG. 2, PPS response of a 190- $\mu$ m-thick quartz plate used for calibration. 


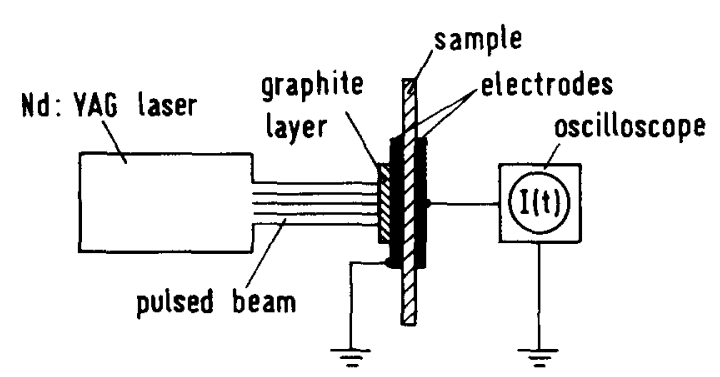

FIG. 3. Experimental setup of the laser-induced pressure-pulse (LIPP) method for two-sided metallized samples.

sound velocity $c$. A detailed description of the LIPP method can be found in the literature. ${ }^{22,26,28}$

\section{Principle of operation}

Upon propagation of a short pressure pulse of duration $\tau$, only a sample layer of thickness $c \tau$ is compressed at a given time $t$. During the following time interval $\Delta t$, this compression zone moves from the location $x=c t$ to the location $x=c(t+\Delta t)$. Thus, the charge on the short-circuited sample electrodes changes from

$$
\frac{Q(t)}{A}=e_{33}^{*}(c t) \frac{v \tau}{s}=e_{33}^{*}(c t) \frac{p \tau}{\rho_{0} c s},
$$

to

$$
\begin{aligned}
& \frac{Q(t+\Delta t)}{A}=e_{33}^{*}[c(t+\Delta t)] \frac{v \tau}{s} \\
& =e_{33}^{*}[c(t+\Delta t)] \frac{p \tau}{\rho_{0} c s} .
\end{aligned}
$$

Therefore, the change $\Delta Q(t)=Q(t+\Delta t)-Q(t)$ of the electrode charge is determined by the change $\Delta \mathrm{e}_{33}^{*}(\mathrm{ct})=\mathrm{e}_{33}^{*}[c(\mathrm{t}+\Delta \mathrm{t})]-\mathrm{e}_{33}^{*}(\mathrm{ct})$ of the apparent piezoelectric coefficient:

$$
\frac{\Delta Q(t)}{A}=\frac{v}{s}-\Delta e_{33}^{*}(c t)=\frac{p \tau}{s \rho_{0} c} \Delta e_{33}^{*}(c t)
$$

Use of $\Delta Q=I \cdot \Delta t$ and $\Delta x=c \cdot \Delta t$, division by $\Delta t$, and transition from differences $\Delta$ to differentials $d$ leads to

$$
I(t)=\frac{A}{s} c v \tau \frac{d e_{33}^{*}}{d x}(c t)=\frac{A}{s} \cdot \frac{p \tau}{\rho_{0}} \cdot \frac{d e_{33}^{*}}{d x}(c t) .
$$

According to Eqs. (2) and (5), a pressure-step experiment reveals the piezoelectricity profile directly, whereas a pressure-pulse measurement discloses the gradient of the piezoelectricity distribution in the sample.

\section{Principle of calibration}

A quartz plate in place of the sample can be utilized to calibrate the LIPP method. As an example, the LIPP response of a $190-\mu \mathrm{m}$-thick two-sided metallized $X$-quartz disk is shown in Fig. 4. The signal indicates the arrival of the LIPP at the quartz surface. Here, the change $\Delta e_{33}^{*}(0)$ of the apparent piezoelectric coefficient is equal to the coefficient $e_{11}$ of $X$-quartz. Therefore, Eq. (4) can be employed to determine the calibration factor $A p \tau /\left(\rho_{0} s\right)$ required for the quantitative evaluation of LIPP results by means of Eq. (5). This factor is directly obtained by dividing $\Delta Q(0)$, which is equal

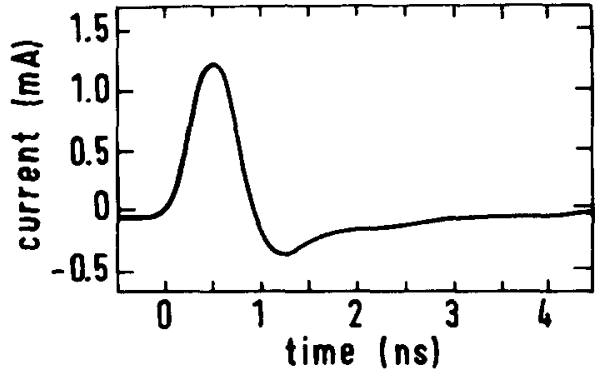

FIG. 4. LIPP response of a $190-\mu \mathrm{m}$-thick quartz plate.

to the area under the curve in Fig. 4, by the piezoelectric coefficient $e_{11}$ and the sound velocity $c_{Q}$ of quartz.

As with the PPS method, the quartz response (Fig. 4) provides an image of the pressure pulse itself. ${ }^{33,34}$ However, the rise and fall times of the signal in Fig. 4 are limited by the bandwidth of the oscilloscope ( $1 \mathrm{GHz}$ ). Thus, the real duration of the LIPP may be even shorter than the signal duration of about $1 \mathrm{~ns}$ shown here. The full width at half maximum (FWHM) of the signal amounts to approximately 0.5 ns, which corresponds to a spatial resolution of less than 1.5 $\mu \mathrm{m}$ in PVDF (sound velocity $c \approx 2.3 \mathrm{~km} / \mathrm{s}$ ). This resolution can, of course, only be realized if the sample capacitance is so small that the time constant of the measuring circuit remains well below $0.5 \mathrm{~ns}$.

In any case, the total charge $\Delta Q(0)$ flowing when the LIPP enters the quartz plate is truly represented by the area under the signal ( $(I d t)$. The term $A p \tau$ in Eq. (5) describes the total mechanical momentum contained in the pressure pulse, and therefore remains constant during the first transit of a LIPP through the sample. The pressure-pulse energy, however, is affected by attentuation in the sample (see below).

The negative part of the signal in Fig. 4 presumably originates from the thermal-stress effects which partly generate the LIPP. These effects produce both compression and rarefaction in the target, whereas the other two main LIPPgenerating mechanisms (ablation and radiation pressure) lead to compression only. The relative contribution of each of these three mechanisms to the LIPP is influenced by the target material and by the laser pulse itself. Almost purely compressional LIPP's can be achieved by optimization of the influencing parameters. ${ }^{27}$

Quantitative evaluation of typical LIPP results obtained with the $190-\mu$ m-thick quartz plate (see e.g., Fig. 4) yields a mechanical momentum $A p \tau$ of approximately $3 \times 10^{-9}$ Ns and thus a pressure amplitude $p$ of about 0.5 $\mathrm{MPa}$. This evaluation is in close agreement with a previous estimate $^{26}$ of $0.6 \mathrm{MPa}$, derived from the known charge density of a Teflon FEP sample. An exact calibration of the LIPP results would require a measurement of the actual laser-pulse amplitude which varies considerably from pulse to pulse. Therefore, only estimates of the absolute ordinates are given with the present LIPP results.

\section{Piezoelectrically generated pressure pulses (PPP's)}

The experimental arrangement of the PPS method (Fig. 1) can also be used to perform pressure-pulse experiments. 


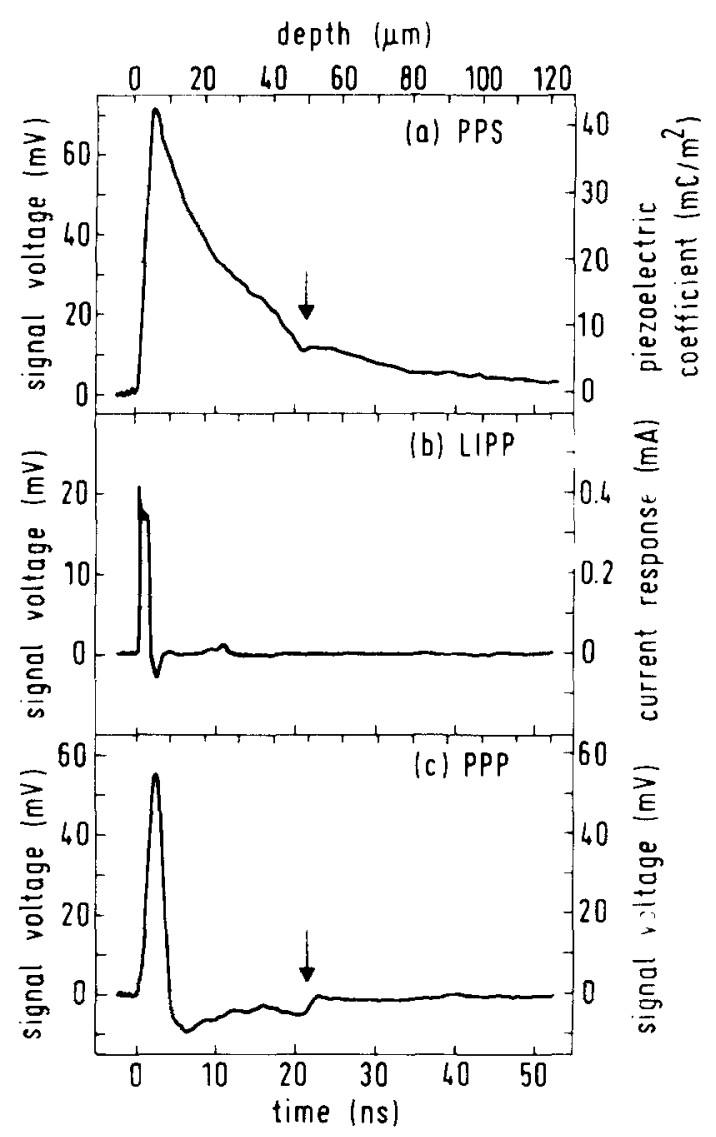

FIG. 5. Responses of a 109- $\mu$ m-thick Dyflor PVDF film measured with (a) the PPS, (b) the LIPP, and (c) the PPP method. The sample was thermally poled $\left(1 \mathrm{~h}\right.$ at $\left.120^{\circ} \mathrm{C}\right)$ with a poling field of about $50 \mathrm{MV} / \mathrm{m}$. Arrows indicate signals caused by reflections at the preamplifier input.

For this purpose, short square pulses of about 1-ns duration are produced by means of the cable-discharge generator (short cable) and are applied to the quartz plate. The principle of operation and the theoretical description of this piezoelectrically generated pressure-pulse (PPP) technique are analogous to those of the LIPP method [Eqs. (4) and (5)]: the PPP response represents the derivative of the respective PPS response. PPP results presented here are preliminary and were not calibrated. The piezoelectric generation of even shorter pressure pulses than those used here is possible and will be attempted in the future.

\section{RESULTS AND DISCUSSSION}

\section{A. High-temperature-poled samples}

The responses of a $109-\mu \mathrm{m}$-thick Dyflor PVDF foil, poled by the thermal method $\left(+5 \mathrm{kV}\right.$ at $120^{\circ} \mathrm{C}$ for $\left.1 \mathrm{~h}\right)$, are shown in Fig. 5. As seen from Fig. 5(a), the piezoelectric activity as measured with the PPS technique rises sharply at the sample surface which was in contact with the positive electrode during poling. The activity gradually decreases with increasing depth and is zero at the other sample surface, whose position is calculated from the known ${ }^{25,26}$ longitudinal sound velocity $c$ in $\operatorname{PVDF}(\approx 2.3 \mathrm{~km} / \mathrm{s})$.

This result agrees well with the reported nonuniformity of the piezo- and pyroelectric activity in thermopoled
PVDF. ${ }^{13-16,23-25}$ Since the sample investigated here was poled with the relatively low field of less than $50 \mathrm{MV} / \mathrm{m}$, it only exhibits piezoelectric activity close to the positive electrode. The present high-resolution technique also revels the shape of the piezoelectricity distribution quite accurately. From such shapes and their dependence on several parameters, a detailed model of the piezoelectric effect in PVDF was derived. ${ }^{23-25}$

Pressure-pulse experiments on the same PVDF sample resulted in the LIPP and PPP responses of Figs. 5(b) and 5(c), respectively. As discussed above, both signals depict the gradient of the piezoelectric activity in the sample. The LIPP response [Fig. $5(\mathrm{~b})$ ] indicates that the piezoelectric activity rises within about the first $4 \mu \mathrm{m}$ from the sample surface, since the LIPP itself (cf. Fig. 4) is much narrower than the signal shown in Fig. 5(b). The detailed shape of the LIPP signal is influenced by the shape of the LIPP itself as shown in Fig. 4. Both the PPS and the PPP response contain a negative peak [marked by arrows in Figs. 5(a) and 5(c)] which stems from a partial relfection of the signal at the preamplifier input. Because of the length of the cable used, the reflected part reaches the preamplifier only after a delay of approximately $20 \mathrm{~ns}$. The delay was chosen to avoid any interference between signal rise and cable reflection.

From the maximum LIPP current of about $0.4 \mathrm{~mA}$ [Fig. 5(b)], the maximum piezoelectric gradient in the sample can be estimated. With Eq. (5), the sample thickness of $109 \mu \mathrm{m}$, the PVDF density of $1.76 \times 10^{3} \mathrm{~kg} / \mathrm{m}^{3}$, and an estimated LIPP momentum of $3 \times 10^{-9} \mathrm{Ns}$ (see above), the maximum gradient becomes $25 \times 10^{3} \mathrm{As} / \mathrm{m}^{3}$. Together with the FWHM of the LIPP peak $(\approx 2 \mu \mathrm{m})$, this maximum gradient results in an estimated maximum piezoelectric coefficient of about $50 \mathrm{mC} / \mathrm{m}^{2}$, in reasonable agreement with the PPS measurement [Fig. 5(a)]

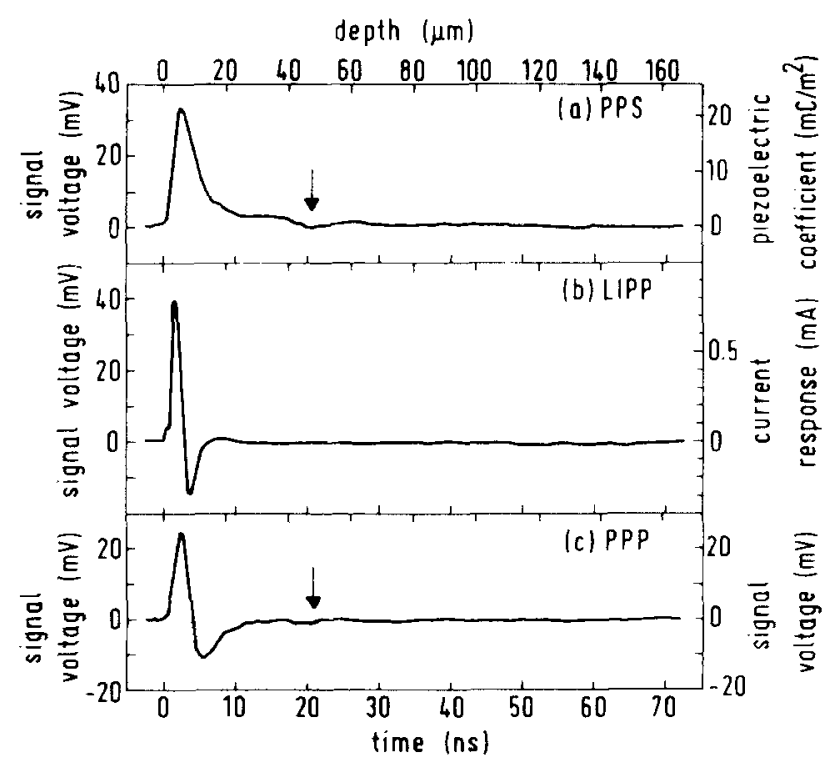

FIG. 6. Responses of a $120-\mu \mathrm{m}$-thick corona-poled Dyflor PVDF film measured with (a) the PPS, (b) the LIPP, and (c) the PPP method through its positively charged surface. Arrows indicate signals from reflections. 


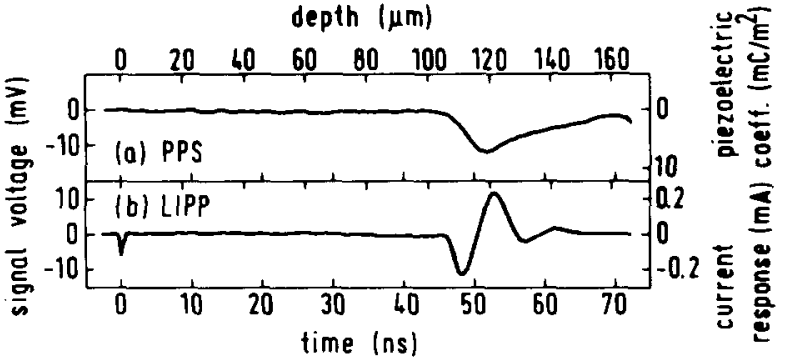

FIG. 7. Responses of a $120-\mu \mathrm{m}$-thick positively corona-poled Dyflor PVDF film measured with (a) the PPS and (b) the LIPP method through the surface opposite to the charged surface. The sample is the same as the one described in Fig. 6.

\section{B. Corona-poled samples}

The PPS, LIPP, and PPP responses of a $120-\mu \mathrm{m}$-thick Dyflor PVDF film are depicted in Figs. 6(a), 6(b), and 6(c), respectively. The sample was charged at room temperature (RT) by means of a positive corona discharge $(+18 \mathrm{kV}, 2$ $\mathrm{min})$. As with the thermopoled sample of the same material, the piezoelectric activity is concentrated near the positively charged surface-a result not reported before. Here again, the peaks caused by reflections in the cable are marked by arrows [Figs. 6(a) and 6(b)].

Figures $7(a)$ and $7(b)$ contain the PPS and LIPP responses of the same sample, respectively, in this case measured through its rear surface. Again, both parts of Fig. 7 show the piezoelectric sample layer which is now reached by the pressure wave only after transit through most of the sample volume. In addition, a small current signal is generated when the LIPP enters the sample [Fig. 7(b)]. This peak could be caused by a small level of piezoelectric activity in the bulk of the sample between the more prominent layer and the

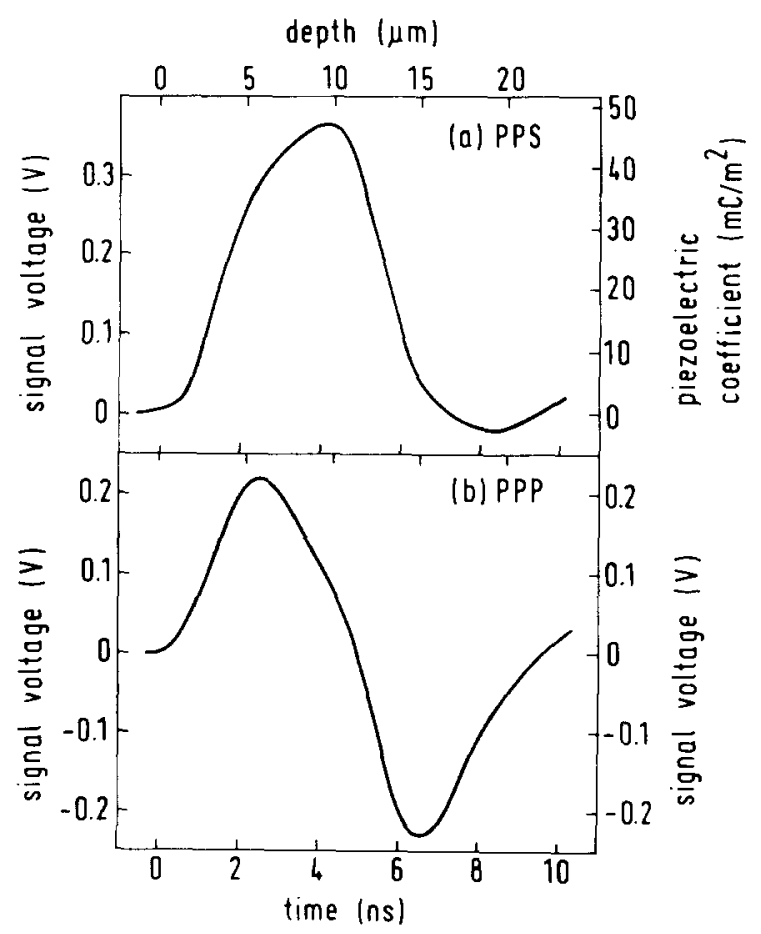

FIG. 8. Responses of an 11- $\mu$ m-thick corona-poled Kureha-KF PVDF film measured with (a) the PPS and (b) the PPP method through its positively charged surface. other surface or by a small amount of charge at the rear electrode.

After reaching the piezoelectric layer, the PPS and the LIPP are partially reflected at the sample surface and traverse the layer again. As a result, a second smaller signal follows in both cases [Figs. 7(a) and 7(b)] immediately after the first signal from the layer. The fact that the signal amplitudes in Fig. 7 are smaller than those in Fig. 6, indicates some attenuation of the pressure waves during transit through the sample. This signal reduction in charged or polarized samples can be used to estimate the ultrasonic attenuation directly from such pressure-wave experiments. ${ }^{35}$ Furthermore, the signals after reflection of the pressure waves in Figs. 7(a) and 7(b) are smaller and wider than those before reflection because of reflection losses and nonparallelity of the sample surfaces. Both effects have to be accounted for in attenuation estimates. ${ }^{35}$

The responses of a positively corona-charged $(+18 \mathrm{kV}$, 2 min, RT) $11-\mu \mathrm{m}$-thick Kureha-KF PVDF film, measured through its front and rear electrodes, are depicted in Figs. 8 and 9, respectively. The PPS and PPP results of Fig. 8, as well as the PPS and LIPP results of Fig. 9, are affected by the capacitance of the very thin sample. The sample capacitance of approximately $50 \mathrm{pF}$ and the impedance of $50 \Omega$, which is used in the high-frequency circuitry of the experiments, result in a time constant of about 2.5 ns. This time constant is of the same order as the transit time of the pressure waves through the sample ( $\approx 4.3 \mathrm{~ns}$ ) and surpasses the rise time of the PPS as well as the duration of the LIPP and the PPP. Therefore, the PPS responses of Figs. 8(a) and 9(a) show a gradual current increase followed by a similar decrease instead of a rectangular shape. Accordingly, the PPP [Fig. 8(b)] and LIPP [Fig. 9(b)] signals exhibit a relatively steep rise and an exponential decay which are influenced by the time constant. Thus, the direct experimental resolution in thinner samples is deteriorated by their larger capacitances.

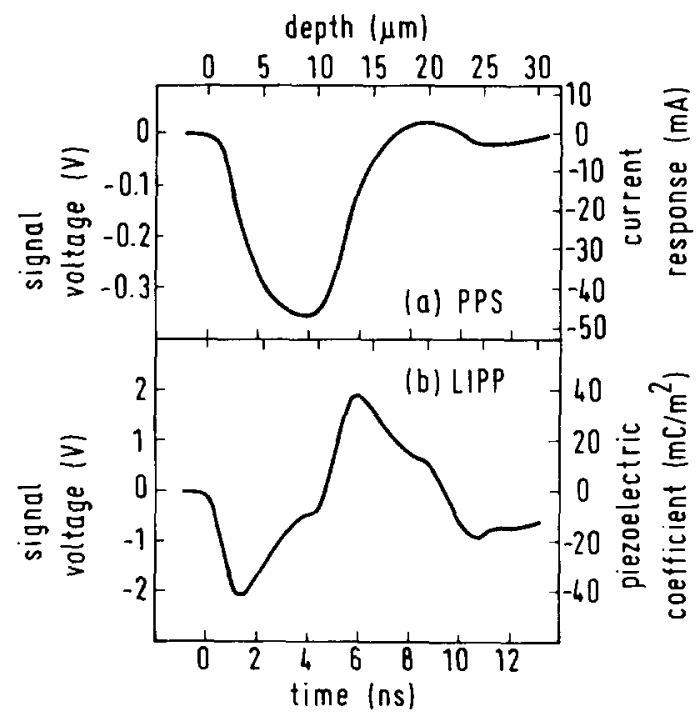

FIG. 9. Responses of an 11- $\mu \mathrm{m}$-thick positively corona-poled Kureha-KF PVDF film measured with (a) the PPS and (b) the LIPP method through the surface opposite to the charged surface. The sample is the same as the one described in Fig. 8. 
The prolongation of the signals caused by the sample capacitance may be avoided by use of a second unpoled foil of the same sample material together with the sample under investigation ${ }^{25,36}$ or by insertion of an air gap between rear surface and rear electrode. Employment of smaller electrodes for the same purpose has the disadvantage of increasing the role of edge effects. All these measures significantly reduce the sample capacitance and the time constant at the expense of the signal amplitude. They thus lead to a better direct experimental resolution.

From the LIPP response shown in Fig. 9(b), the maximum piezoelectric coefficient in the $11-\mu \mathrm{m}$-thick sample is estimated to be about $50 \mathrm{mC} / \mathrm{m}^{2}$, in close agreement with the PPS result of Fig. 9(a). The four current responses of the corona-poled Kureha-KF sample (Figs. 8 and 9) lead to the conclusion that the $11-\mu \mathrm{m}$-thick foil is poled throughout its volume, since all deviations from a rectangular polarization profile can be explained with the long time constant of this sample. In all three samples described here, the maximum piezoelectric coefficient is of about the same size. Thus the poling field across the piezoelectric layer in the thick samples (Figs. 5-7) must have been of the same order as the field across the thin specimen (Figs. 8and 9). This result can only be explained in terms of charge injection into the sample volume during poling in agreement with earlier findings. ${ }^{23-25}$

\section{CONCLUSIONS}

It was demonstrated that the two recently developed high-resolution pressure-wave methods, based on piezoelectric and laser-pulse generation of pressure discontinuities, produce comparable results when applied to piezoelectric PVDF samples. The pressure-step method (rise time $\approx 1 \mathrm{~ns}$ ) gives a direct image of the piezoelectricity profile, whereas the pressure-pulse techniques (pulse widths $>0.5 \mathrm{~ns}$ ) reveal the gradient of the distribution. The differential distributions measured with the latter methods provide an even better resolution than the profiles obtained with the former technique. The pressure amplitude of the LIPP's is of the order of $0.5 \mathrm{MPa}$ (see above), whereas that of the PPS's and PPP's is at least ten times smaller. ${ }^{25}$ Therefore, often a preamplifier is not required for LIPP measurements (see above), and the signal-to-noise ratio is relatively high. However, the much better reproducibility of the amplitude of the PPS's and PPP's allows for the use of sampling and signalaveraging techniques which improve the signal-to-noise ratio. Thus, the presented experimental methods complement each other in several aspects. In principle, deconvolution techniques can be used with all three methods to further improve the resolution. However, the presently possible direct experimental resolution is sufficient for most applications of the new methods.

It was confirmed that the piezoelectric activity of thermopoled PVDF samples is confined to a layer close to the positively biased electrode if relatively low poling fields are used. ${ }^{13-16,23-25}$ The same effect was found for the first time on positively corona-poled PVDF foils. Higher poling fields lead to a more or less uniform distribution of the piezoelectric activity in a corona-poled sample. ${ }^{36}$ Previously found piezoelectricity distributions in $\mathrm{PVDF}^{23-25}$ are supplemented by the present findings.

\section{ACKNOWLEDGMENTS}

The authors are grateful to Dr. H. von Seggern for stimulating discussions, to Dr. D A. Berkley for valuable comments on the manuscript, and to W. Burger and R. Kubli for sample preparation. The LIPP experiments were carried out using the high-energy laser facility of the Electronics Research Laboratory at Bell Laboratories, Holmdel, New Jersey, while the PPS and PPP measurements were performed at theUniversity of Stuttgart. Both collaborating groups are indebted to the Deutsche Forschungsgemeinschaft for financial support.

${ }^{1}$ H. Kawai, Jpn. J. Appl. Phys. 8, 975 (1969).

${ }^{2}$ R. G. Kepler, Annu. Rev. Phys. Chem. 29, 497 (1978).

${ }^{3}$ M. G. Broadhurst and G. T. Davis, in Electrets, Vol. 33 of Topics in Applied Physics, edited by G. M. Sessler (Springer, Berlin/New York, 1980), Chap. 5, pp. 285-319; G. M. Sessler and J. E. West, ibid., Chap. 7, pp. 347-381.

${ }^{4}$ R. G. Kepler and R. A. Anderson, CRC Crit. Rev. Solid State Mater. Sci. 9, 399 (1980).

${ }^{5}$ E. Fukada and T. Furukawa, Ultrasonics 19, 31 (1981).

${ }^{6} D$. K. Das-Gupta, Ferroelectrics 33, $75(1981)$.

${ }^{7}$ G. M. Sessler, J. Acoust. Soc. Am. 70, 1596 (1981).

${ }^{8}$ A. J. Lovinger, in Developments in Crystalline Polymers-1, edited by D. C. Bassett (Applied Science, London/New Jersey, 1982), Chap. 5, pp. 195273.

${ }^{9}$ M. A. Marcus, Ferroelectrics 40, 29 (1982).

${ }^{10} \mathrm{G}$. M. Sessler, in Electrical Properties of Polymers, edited by D. A. Seanor (Academic, New York, 1982), Chap. 6, pp. 241-284.

${ }^{11} \mathrm{Y}$. Wada, in Electronic Properties of Polymers, edited by J. Mort and G Pfister (Wiley, New York, 1982), Chap. 4, pp. 109-160.

${ }^{12}$ S. B. Lang, Ferroelectrics 32, 191 (1981); 34, 239 (1981); 45, 283 (1982); 46, 51 (1982); 47, 259 (1983).

${ }^{13}$ R. J. Phelan, Jr., R. L. Peterson, C. A. Hamilton, and G. W. Day, Ferroelectrics 7, 375 (1974).

${ }^{14}$ H. Sussner and K. Dransfeld, J. Polym. Sci. Polym. Phys. Ed. 16, 529 (1978).

${ }^{15}$ M. A. Marcus, Ferroelectrics 32, $149(1981)$.

${ }^{16}$ M. A. Marcus, J. Appl. Phys. 52, $6273(1981)$.

${ }^{17}$ R. E. Collins, Rev. Sci. Instrum. 48, 83 (1977).

${ }^{18}$ P. Laurenceau, G. Dreyfus, and J. Lewiner, Phys. Rev. Lett. 38, 46 (1977).

${ }^{19}$ A. G. Rozno and V. V. Gromov, Pis'ma Zh. Tekh. Fiz. 5, 648(1979) [Sov. Tech. Phys. Lett. 5, 266 (1979)].

${ }^{20} A$. Migliori and J. D. Thompson, J. Appl. Phys. 51, 479 (1980).

${ }^{21}$ C. Alquié, G. Dreyfus, and J. Lewiner, Phys. Rev. Lett. 47, 1483 (1981).

${ }^{22}$ G. M. Sessler, J. E. West, and R. Gerhard, Polym. Bull. 9, 109 (1981); Phys. Rev.Lett. 48, 563 (1982).

${ }^{23}$ W. Eisenmenger and M.Haardt, Solid State Commun. 41, 917 (1982).

${ }^{24}$ M. Haardt and W. Eisenmenger, in 1982 Annual Report Conference on Electrical Insulation and Dielectric Phenomena (IEEE Service Center, Piscataway, 1982), pp. 46-51; W. Eisenmenger, M. Haardt, and K. Holdik, ibid., pp. 52-57.

${ }^{25} \mathrm{M}$. Haardt, $\mathrm{Ph} . \mathrm{D}$. thesis, University of Stuttgart, 1982.

${ }^{26} \mathrm{G}$. M. Sessler, J. E. West, R. Gerhard-Multhaupt, and H. von Seggern, IEEE Trans. Nucl. Sci. NS-29, 1644 (1982).

${ }^{27} \mathrm{C}$. Alquié, J. Lewiner, and G. Dreyfus, J. Phys. (Paris) Lett. 44, L-171 (1983).

${ }^{28}$ R. Gerhard-Multhaupt, Phys. Rev. B 27, 2494 (1983).

${ }^{29}$ R. Gerhard-Multhaupt, M. Haardt, W. Eisenmenger, and G. M. Sessler, J. Phys. D 16, 2247 (1983). 
${ }^{30} \mathrm{P}$. D. Southgate, Appl. Phys. Lett. 28, 250 (1976).

${ }^{31}$ W. Burger, Dipl.-Phys. thesis, University of Stuttgart, 1983.

${ }^{32}$ D. A. Berlincourt, D. R. Curran, and H. Jaffe, in Physical Acoustics, edited by W. P. Mason (Academic, New York 1964), Vol. 1, Part A, Chap. 3, pp. 169-270.

${ }^{33}$ W. Eisenmenger, Acoustica 12, 165 (1962).
${ }^{34}$ E. H. Jacobsen, J. Acoust. Soc. Am. 32, 949 (1960).

${ }^{35}$ G. M. Sessler, R. Gerhard-Multhaupt, and J. E. West, in Proceedings of the 11th International Congress on Acoustics, Paris 1983, Rev. d'acoustique, hors série Vol. 2, pp. 195-198 (1983).

${ }^{36}$ W. Eisenmenger, M. Haardt, and K. Holdik, Verh. Dtsch. Phys. Ges. (VI) 18, $31(1983)$ 